\title{
Influência de corantes na estabilidade oxidativa de amostras de biodiesel
}

\section{Influence of dyes in oxidative stability of samples of biodiesel}

\author{
Gemima dos Santos ${ }^{1 *}$, Magno Aparecido Trindade ${ }^{2}$, Valdir de Souza Ferreira ${ }^{1}$, Lincoln \\ Carlos Silva de Oliveira ${ }^{1}$, Paulo César Cavalcante Vila Nova ${ }^{1}$ e Eduardo José de Arruda ${ }^{2}$
}

\begin{abstract}
${ }^{1}$ Departamento de Química, Universidade Federal de Mato Grosso do Sul, 79074-460 Campo Grande-MS, Brasil. ${ }^{2}$ Faculdade de Ciências Exatas e Tecnologia/Química, Universidade Federal da Grande Dourados, 79804-970, Dourados-MS, Brasil.
\end{abstract}

\begin{abstract}
The feasibility of using the dyequinizarin (QNZ) on the oxidative stability of soybean biodiesel samples was studied in this work. The paper presents parameters indicative of the oxidation of biodiesel under thermal degradation through measurements of its acid value, peroxide and conjugated dienes and trienes. The study aimed to evaluate the behavior/performance antioxidant of the dyequinizarin (QNZ) along with the antioxidant tert -

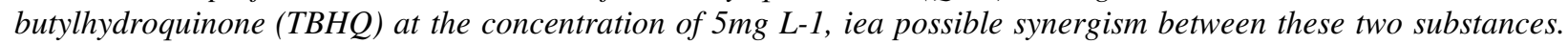
The samples were characterized by physicochemical analyzes and spectrophotometric UV/Vis(200-400 nm). The analysis of the acid number and peroxide were consistent with the absorbance at 232and 270nm, for in both analyzes were detected increase in the oxidation of biodiesel samples throughout storage period and/or thermal degradation.
\end{abstract}

Key-words: Biodiesel, dye, quinizarin, antioxidants, tert-butylhydroquinone, oxidative stability

\section{INTRODUÇÃO}

O aumento no consumo de combustíveis derivados do petróleo tem sido decorrente do crescimento acelerado no mercado mundial de veículos automotores. Neste cenário, consequentemente há um crescente interesse por fontes alternativas de energia a fim de minimizar problemas potenciais devido ao uso de combustíveis fósseis, tais como a dependência do fornecimento de petróleo, o esgotamento futuro dessas fontes energéticas, além dos impactos ambientais e climáticos devido aos poluentes resultantes da combustão dos derivados de petróleo e seus problemas poluentes (Knothe, 2005).

O biodiesel é uma fonte alternativa de energia renovável com potencial aplicação na substituição total ou parcial do óleo diesel, principalmente em motores de ignição onde não há necessidade de adaptações do motor ao combustível ao novo sistema (Ferreira e Oliveira, 2005).O biodiesel,diferentemente dos óleos combustíveis derivados do petróleo que são estáveis a altas temperaturas, mesmo na presença de oxigênio, são instáveis e apresentam grandes tendências a degradação oxidativa (Ferrari, 2009).Estas reações de oxidação são mais relevantes em altas temperaturas, onde podem ocorrer reações complementares de decomposição térmica, formação de compostos poliméricos e outros produtos, principalmente por reações de condensação (Mazzeto, 2009).

De acordo com a literatura, Monteiro (2008) e Quintella (2009) descrevem o processo oxidativo do biodiesel, bem como outras alterações nas propriedades físico-químicas que são decorrentes da exposição à luz, ao ar, a umidade, metais, calor e tempode armazenagem. No entanto, oprocesso de degradação pode ser retardado pelo uso de agentes externos, tais como os antioxidantese/ou

Author for correspondence: gemima100@yahoo.com.br 
agentes complexantes/quelantes que são empregados especialmente para aumentar a estabilidade do biodiesel (Filho, 2010).

No biodiesel, a ação dos agentes antioxidantes é capturar espécies radicalares livres formadas durante os processos oxidativos, interrompendo a reação em cadeia de degradação do combustível. Estes antioxidantes em geral são substâncias contendo grupos aminas ou hidroxilas com elétrons de valência livres que são mais eletroativos que os ácidos graxos insaturados na estrutura do biodiesel após a síntese por esterificação.Substâncias com características antioxidantes tais como, t-butilhidroquinona (TBHQ), hidróxi-tolueno butilado (BHT), $\alpha$ tocoferol, entre outras moléculas são propostas, e, têm sido empregados satisfatoriamente como aditivosretardantes do efeito de oxidação do biodiesel (Monyem, 2001; Filho, 2010).

Assim, com a necessidade de tornar o biodiesel um combustível mais estável ao uso e armazenamento, vários estudos vem sendo realizados empregando antioxidantes como forma de aumentar à estabilidade dos combustíveis a estocagem (Freitas, 2009). O uso de corantes é bastante promissor para adição ao biodiesel, para minimização ou controle dos processos oxidativos e até para identificação do produto/fabricante ao consumidor. Na mistura dessecorante com agentes antioxidantes, poderá haver efeito de sinergismo ou potencialização dos efeitos protetivos, que ocorre quando a sua mistura produz um efeito maior que a soma das atividades dos antioxidantes/protetivas testadas individualmente (Shay, 1993; Dabdoub, 2009).

O efeito antioxidante do corante QZN está relacionado com a quantidade de grupos hidroxilas presentes na molécula e a facilidade com que estes se oxidam, podendo também interagir com a molécula do antioxidante, i.e TBHQ e alterar as distribuições eletrônicas de carga, potencializando a ação antioxidante (Karavalakis \& Stournas, 2010). Assim, considerando-se a presença no corante de grupos substituintes com átomos doadores ou aceitadores de elétrons, verifica-se que esta molécula é semelhante às moléculas dos antioxidantes de uso comum em biodiesel. Esta similaridade pode ser explorada para o desenvolvimento de um novo sistema de aditivos com vistas a auxiliar ou substituir os antioxidantes convencionais a fim de garantir maior estabilidade ao biodiesel.
O corante quinizarina mostrado na Figura 1 possuio grupo antraquinona, que a princípio tem estrutura favorável para agir/atuar como antioxidante em função da sua facilidade de se oxidar em atmosfera com oxigênio, servindo como molécula de sacrifício, e potencializando a ação antioxidante e oferecendo maior proteção ao biodiesel.

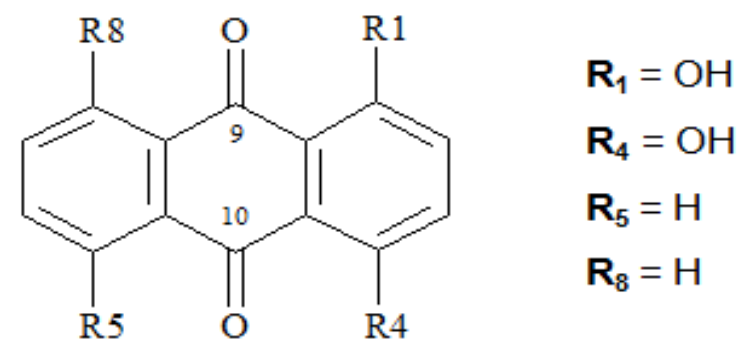

Figura 1-Estrutura molecular do corante quinizarina (QNZ).

O presente trabalho tem como objetivo estudar o efeito/ação protetiva e sinérgica do corante quinizarina adicionado individualmente e/ou juntamente com o antioxidante terc-butilhidroquinona (TBHQ) na estabilização do biodiesel de soja sob estocagem.

\section{MATERIAL E MÉTODOS Amostra de biodiesel}

A obtenção do biodiesel foi realizada pelo método de transesterificação de acordo com a proporção de álcool/óleo utilizado nos experimentos de 6/1, a $1 \%$ de catalisador básico $\mathrm{NaOH}$ P.A. a temperatura de $60^{\circ} \mathrm{C}$ e tempo de reação de 60 minutos (Knothe, 2007).

\section{Soluções}

Todas as soluções usadas na determinação dos índices de acidez (IA) e peróxido (IP)foram preparadas no momento do uso, com o emprego de água ultrapura Milli-Q. Esta água foi utilizada para a preparação das seguintes soluções de concentrações:

-Solução aquosa de tiossulfato de sódio $\left(\mathrm{Na}_{2} \mathrm{~S}_{2} \mathrm{O}_{3} .5 \mathrm{H}_{2} \mathrm{O}\right)$ 0,02 mol. $\mathrm{L}^{-1}$;

-Solução aquosa saturada de iodeto de potássio (KI);

-Solução de indicador amido 1\%;

-Solução aquosa de Hidróxido de Sódio $(\mathrm{NaOH})$ 0,01 mol. $\mathrm{L}^{-1}$. 


\section{Preparação das amostras}

A aditivação das amostras de biodiesel de soja com o corante quinizarina (QNZ) e com o antioxidante (TBHQ) foram feitas ambas na concentração de 5mg. $\mathrm{L}^{-1}$.

A amostra contendo tanto o corante QNZ quanto o antioxidante TBHQ (relação mássica 1:1) foi obtida através da adição de $5 \mathrm{mg} . \mathrm{L}^{-1}$ de QNZ e 5 mg. $L^{-1}$ de TBHQ em balão de $250 \mathrm{~mL}$ sob agitação. Em seguida a amostra foi submetida ao mesmo processo de homogeneização descrito acima.

\section{Teste de oxidação em estufa}

Após a aditivação das amostras de biodiesel de soja, as mesmas foram colocadas em uma estufa com temperatura de $65^{\circ} \mathrm{C}$, durante um período de 336 horas, para que sofressem extensa degradação térmica.

Para cada intervalo de tempo de 48 horas foram retiradas alíquotas de biodiesel em que foram determinados os índices de acidez (IA), peróxido (IP) e medidas de absorbância por espectrofotometria UV/Vis no intervalo de 200 a $400 \mathrm{~nm}$ para avaliação da presença e intensidade dos grupos resultantes da degradação térmica.

\section{Índice de acidez (IA)}

Os ensaios de índice de acidez (IA) foram realizados de acordo com as normas da Associação Brasileira de Normas Técnicas (ABNT) indicadas pela Resolução $\mathrm{n}^{\circ} 42$ da Agência Nacional de Petróleo, Gás Natural e Biocombustíveis (ANP) (Falcone, 2007).

\section{Índice de peróxido (IP)}

Os ensaios de peróxido foram realizados de acordo com (AOCS, 1990).

\section{Análise por espectroscopia de absorção eletrônica $(\mathrm{UV} / \mathrm{Vis})$}

As amostras de biodiesel aditivadas foram analisadas por espectroscopia no visível e ultravioleta, com um espectrofotômetro UV/Vis. Hitachi, modelo U-3000, com a varredura dos espectros de 200 a $400 \mathrm{~nm}$ em cubetas de quartzo de $1 \mathrm{~cm}$ de caminho óptico. As amostras foram diluídas em diclorometano, na proporção de 1:1000segundo a metodologia proposta por Monteiro (2008).

\section{RESULTADOS E DISCUSSÕES}

O índice de acidez (IA)revela o estado de conservação do óleo ou do biodiesel. É definido como a massa $(\mathrm{mg})$ de hidróxido de potássio necessária para neutralizar os ácidos livres em 1,0g (uma grama) de amostra, ou seja, quanto maior o índice de acidez, maior a oxidação do óleo ou biodiesel.

A Figura 2mostraa susceptibilidade do biodiesel à oxidação sob ação de fontes de calor e atmosfera de oxigênio. Pode-se observar que um elevado aumento no índice de acidez (IA) com o passar do tempo na amostra de biodiesel não aditivada (isenta da aditivação), com antioxidantes e/ou corantes. Esta amostra foi a que apresentou maior aumento no índice de acidez no período de estudo, com valor de aproximadamente $50 \mathrm{mg}$ de $\mathrm{KOH}$ por grama da amostra.

Já a amostra de biodiesel aditivada somente com o corante QNZ e a amostra aditivada com o antioxidante TBHQ comportaram-se de modo similar. Estas foram às amostras as que atingiram o um menor índice de oxidação no período do estudo. Estas amostras representam as que obtiveram melhor eficiência na estabilização oxidativa do biodiesel sob as condições de degradação térmica e nas condições estudadas.

A amostra aditivada com QNZ e TBHQ não se comportou de maneira satisfatória, pois, possivelmente,pode ter ocorrido interação molecular e bloqueio/indisposição dos grupos hidroxilas, responsáveis pelos efeitos protetivos e antioxidantes no biodiesel (Karavalakis \& Stournas, 2010). Este fato fez com que essa amostra aditivada com QZN e TBHQ (QZN+TBHQ) fosse classificada como a de menor índice de eficiência quanto à atividade antioxidante frente à oxidação por via térmica do biodiesel.

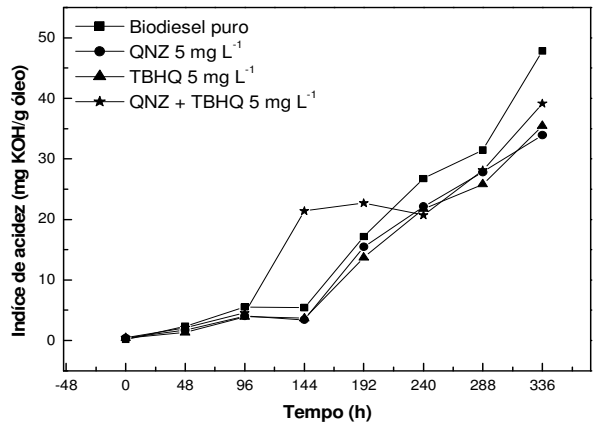

Figura 2- Índice de acidez (IA) da amostra de biodiesel de soja puro (BS), amostra aditivada com corante QNZ $\left(5 \mathrm{mg} . \mathrm{L}^{-1}\right)$, amostra aditivada com antioxidante TBHQ(5 
mg. $\left.\mathrm{L}^{-1}\right)$ e amostra aditivada com mistura 1:1 QNZ e TBHQ (QZN+TBHQ), (5 mg.L $\left.\mathrm{L}^{1}\right)$ submetidas à degradação térmica em estufa a $65^{\circ} \mathrm{C}$ por 336 horas.

O gráfico da Figura 3 demonstra que a amostra de biodiesel que não fora aditivado é a que oxida mais rapidamente. Pode-se observar também que o biodiesel acrescido apenas de QNZ na concentração de $5 \mathrm{mg} . \mathrm{L}^{-1}$ apresentou o menor aumento no índice de peróxido. Isto se deve as ligações de hidrogênio intramoleculares que aprisionam o par de elétrons não ligantes das carbonilas, contribuindo para a melhor deslocalização de elétrons e estabilização dos radicais livres (Massucatto, 2009).

O comportamento da amostra contendo o corante QNZ foi o que mostrou melhor resultado e maior eficiência na estabilização/proteção oxidativa do biodiesel em comparação a amostra não aditivada. Já a amostra aditivada contendo o antioxidante TBHQ obteve a segunda melhor eficiência protetiva. Entretanto, a mistura com corante (QZN) e antioxidante TBHQ (QZN/TBHQ) foi à amostra que teve resultados menos satisfatórios, mostrando-se inviável, nestas concentrações e condições de estudo, para o objetivo em questão.

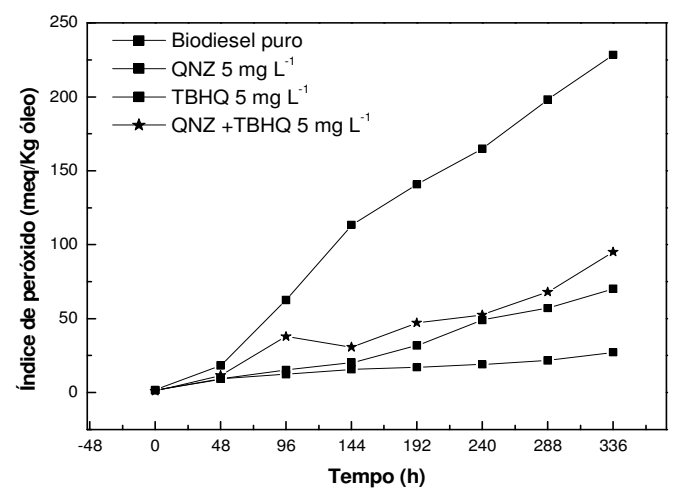

Figura 3-Índice de peróxido (IP) do biodiesel de soja puro (BS), amostra aditivada com corante QNZ (5mg. $\left.\mathrm{L}^{-1}\right)$, amostra com antioxidante TBHQ (5 mg. $\mathrm{L}^{1}$ ) e amostra aditivada com a mistura $1: 1$ QNZ e TBHQ $\left(5 \mathrm{mg} . \mathrm{L}^{-1}\right)$ submetidas à degradação térmica em estufa a $65^{\circ} \mathrm{C}$ por 336 horas.

Os resultados obtidos para absorbância específica no UV-Vis a 232 e 270nm são apresentados nas Figuras 4 e 5, respectivamente.

De acordo com a Figura 4,observa-se o mesmo comportamento apresentado nos estudos anteriores. Pode-se observar um crescente aumento no índice de oxidação das amostras de biodiesel de soja (BS) e aditivados (QZN, TBHQ e QZN+TBHQ) com o aumento do tempo de degradação térmica.

A amostra de biodiesel pura (BS) foi a que mais sofreu o processo oxidativo. A amostra apresentou valores de absorbâncias crescentes durante o período de estudo, o que indica a formação de insaturações (dienos) que são produtos da oxidação do biodiesel.

A amostra contendo o corante QNZ foi a que apresentou menor aumento nas absorbâncias durante o período de estudo de degradação térmica, seguida pela amostra contendo o antioxidante TBHQ. Entretanto, pode-se observar que estas duas amostras tiveram comportamentos muito similares até aproximadamente 192 horas de degradação. Após este tempo de degradação, a amostra contendo o antioxidante TBHQ teve um pequeno aumento nos seus valores de absorbâncias. Já a amostra contendo a adição das duas substâncias em estudo (QZN+TBHQ), foi a que menos se destacou frente à estabilização contra atividade oxidativa por via térmica.

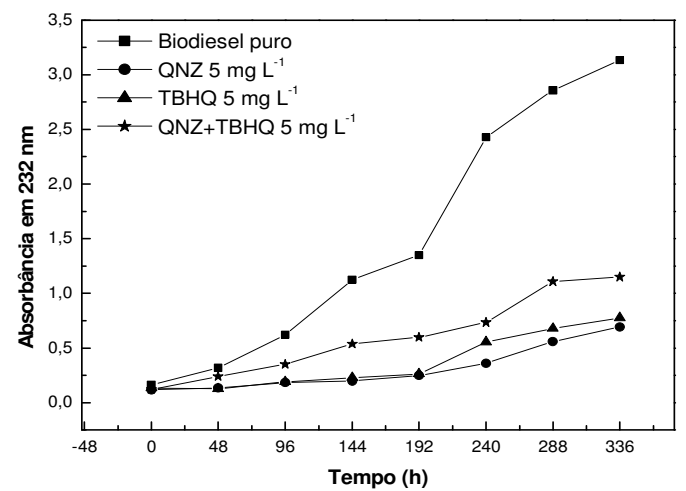

Figura 4-Absorbância em 232nm das amostras de biodiesel de soja puro (BS), amostra aditivada com corante QNZ (5 mg. $\left.\mathrm{L}^{-1}\right)$, amostra aditivada com antioxidante TBHQ (5 mg. $\left.\mathrm{L}^{-1}\right)$ e amostra com a mistura (com o sinergismo entre) QNZ e TBHQ (QZN+TBHQ) (5 mg.. $\left.\mathrm{L}^{-1}\right)$ submetidas à degradação térmica em estufa a $65^{\circ} \mathrm{C}$ por 336 horas.

No gráfico da Figura 5,verifica-se praticamente o mesmo comportamento que observados para os estudos em 232nm, com uma pequena diferença de que, o aumento da absorbância nas amostras durante o período de estudo e este comportamento pode estar está relacionado à formação de insaturações (trienos). Este resultado está coerente com o ganho proporcional de oxigênio e à 
formação de peróxidos durante o estágio da oxidação térmica (Filho, 2010).

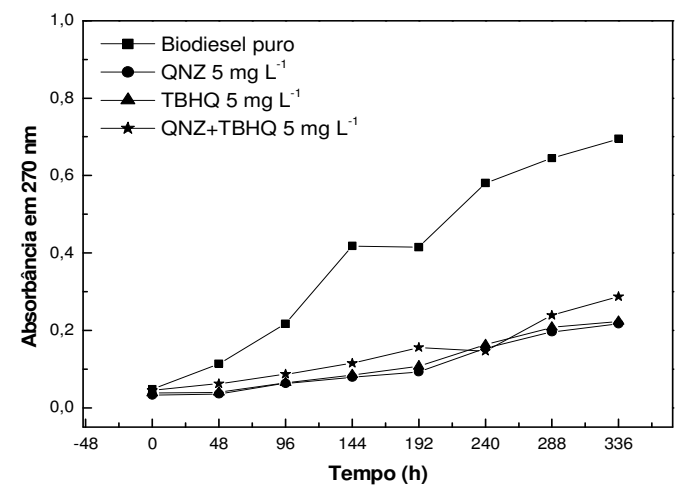

Figura 5-Absorbância em $270 \mathrm{~nm}$ das amostras de biodiesel de soja puro (BS), amostra aditivada com corante QNZ (5 mg. $\left.\mathrm{L}^{-1}\right)$, amostra aditivada com antioxidante TBHQ (5 mg. $\left.\mathrm{L}^{-1}\right)$ e amostra aditivada com a mistura 1:1 QNZ e TBHQ (QZN+TBHQ) (5 mg.L $\left.{ }^{1}\right)$ submetidas à degradação térmica em estufa a $65^{\circ} \mathrm{C}$ por 336 horas.

Vários trabalhos da literatura mostraram a eficiência do antioxidante TBHQ frente à atividade oxidativa de amostras de biodiesel, produzido a partir de diversos óleos vegetais (Ferrari, 2009; Domingos, 2007; Liang, 2006). Este antioxidante foi determinado com sucesso em amostras de biodiesel na concentração de 667 mg.L $\mathrm{L}^{-1}$ (Tang et al., 2010).

A susceptibilidade do biodiesel a oxidação é bem conhecida e a sua aditivação utilizando misturas sinérgicas potencializadas pode permitir ganhos econômicos, e em até alguns casos o aumento da eficiência da queima do biodiesel pela quantidade de oxigênio disponível nas moléculas dos antioxidantes utilizados (Caires, 2012; Soares, 2010).Por isso, foi avaliada a susceptibilidade do biodiesel à oxidação quando aditivado com duas substâncias, ou seja, avaliar/observar um possível sinergismo entre o corante QNZ e o antioxidante TBHQ, ambos na mesma concentração de $5 \mathrm{mg} \mathrm{L}^{-1}$. Juntamente com esse estudo, também foi avaliado o comportamento individual dessas substâncias nas amostras de biodiesel.

\section{CONCLUSÕES}

A eficiência de antioxidante TBHQ comparada com o corante QNZ ao longo do processo de degradação térmica foi considerada inferior. Este resultado pode estar relacionado à baixa concentração e/ou a relação mássica utilizada de
TBHQ (5 mg. $\mathrm{L}^{-1}$ ), uma vez que a determinação com sucesso para obtenção do efeito protetivo na literatura foi determinada na concentração de 667 de $\mathrm{mg} \cdot \mathrm{L}^{-1}$. Esta concentração é $133 \mathrm{x}$ maior que a concentração avaliada.

A intenção de formular uma mistura aditivada com melhor efeito protetivo/antioxidante frente à oxidação do biodiesel não foi possível, pois não ocorreu a potencialização/sinergismo esperada entre o corante QZN e TBHQ.

Nestas condições de concentração e relação mássica uma inviabilidade a mistura proposta para o objetivo em questão. A ação do corante e antioxidante não pode ser observado.

O comportamento do corante QNZ foi satisfatório, no qual mostrou melhor desempenho protetivo para o objetivo do trabalho.

\section{RESUMO}

A viabilidade do uso do corante Quinizarina (QNZ) na estabilidade oxidativa de amostras de biodiesel de soja foi estudada neste trabalho. O trabalho apresenta parâmetros indicativos da oxidação de amostras de biodiesel submetidas à degradação térmica através de determinações do índice de acidez (IA), peróxido (IP) e análise de insaturações por UV-Vis para dienos e trienos conjugados na estrutura da molécula do éster (biodiesel). O estudo teve como objetivo principal avaliar o comportamento de amostras de biodiesel de soja (BS) e de amostras aditivadas (QZN, TBHQ e QZN+TBHQ) na mistura mássica de 1:1 na concentração de $5 \mathrm{mg} . \mathrm{L}^{-1}$. Buscou-se na análise uma possível sinergia a partir dos efeitos protetivos/antioxidantes das duas substâncias, QZN e TBHQ. As amostras de biodiesel de soja (BS) e aditivadas foram avaliadas e caracterizadas mediante análises físico-químicas e espectrofotométricas UV/Vis,no intervalo de 200 a 400nm. As análises do índice de acidez (IA) e peróxido (IP) foram compatíveis com as absorbâncias em $232 \mathrm{~nm}$ e $270 \mathrm{~nm}$, pois em ambas as análises foram detectados aumentos no grau de oxidação das amostras de biodiesel de soja puro (BS) ou aditivados(QZN, TBHQ e QZN+TBHQ) ao longo do período de estocagem.

Palavras-chave: Biodiesel, corante, QZN, antioxidante, TBHQ, estabilidade oxidativa

\section{AGRADECIMENTOS}

A CAPES e CNPq pelo apoio financeiro.

\section{REFERÊNCIAS}

Caires, A. R. L.; Lima, V. S.; Oliveira, S. L. (2012), Quantification of biodiesel content in diesel/biodiesel blends byfluorescence spectroscopy: Evaluation of the dependence on 
biodieselfeedstock. Renewable Energy, 46, 137140.

Dabdoub, M.J.;Bronzel, J.L.; Rampin, M.A. (2009),Biodiesel: visão crítica do status atual e perspectivas na academia e na indústria. Química Nova, 32, 776-792.

Domingos, A. K.; Saad, E. B., Vechiatto, W. W. D.; Wilhelm, H. M.; Ramos, L. P. (2007), The influence of BHA, BHT and TBHQ on the oxidation stability of soybean oil ethyl esters (Biodiesel).

JournaloftheBrazilianChemicalSociety, 18, 416423.

Falcone, A. A. Avaliação da oxidação do biodiesel etílico de milho por meio de técnicas espectroscópias. Dissertação (Mestrado em Química) - Universidade Federal da Paraíba, 2007.

Ferrari, R. A. e Souza, W.L. (2009), Avaliação da estabilidade oxidativa de biodiesel de óleo de girassol com antioxidantes. Química Nova, 32, 106-111.

Ferrari, R. A.; Oliveira, V. S.; Scarbio, A. (2005),Biodiesel de soja-taxa de conversão em ésteres etílicos, caracterização físico-química e consumo em gerador de energia. Química Nova, 28, 19-23.

Filho, M. G. R. Cardanol e eugenol modificados uso como antioxidantes no controle do processo oxidativo do biodiesel etílico de algodão. Tese (Doutorado em Química) -Universidade Federal da Paraíba, 2010.

Freitas, R. C. Biodiesel: O papel do estado regulador e formulador de políticas públicas. Dissertação (Mestrado em Engenharia de Transportes) -Universidade Federal do Rio de Janeiro, 2009.

Karavalakis, G. eStournas, S. (2010), Impact of antioxidant additives on the oxidation stability of diesel/biodiesel blends. Energy Fuels, 24, 36823686.

Knothe, G.; Gerpen, J.V.; Krahl, J. (2005), The biodiesel handbook. Illinois: AOCS Press, 303p.

Knothe, G. H. (2007),Some aspects of biodiesel oxidative stability. Fuel Processing Technology,88, 677-699.

Knothe, G.; Gerpen, J. V.; Krahl, J.; Ramos, L. P. (2006),Manual de Biodiesel, 1. Ed. São Paulo: Editora Blucher.

Liang, Y. C.; May, C. Y.; Foon, C. S.; Ngan, M. A.; Hock, C. C.; Basiron, Y. (2006), The effect of natural and synthetic antioxidants on the oxidative stability of palm diesel. Fuel, 85, 867-870.

Massucatto, D. (2009), Investigação da cinética de lipase através da espectroscopia de fluorescência. Dissertação (Mestrado em Ciências) Universidade de São Paulo, 2009.

Mazzeto, S.E.;Lomonaco, D.; Mele, G. (2009), Óleo da castanha de caju: oportunidades e desafios no contexto do desenvolvimento e sustentabilidade industrial. Química Nova, 32, 732-741.

Monteiro, M. R.; Ambrozini, A. R. P., Lião, L. M.; Ferreira, A. G. (2008),Criticalreviewonanalyticalmethods for biodiesel characterization. Talanta, 77, 593-605.

Monyem, A. e Van Gerpen, J.H. (2001), The effect of biodiesel oxidation on engine performance and emissions. BiomassandBioenergy,20, 317-325.

Quintella, C.M.; Teixeira, L. S. G.; Korn, M. G. A.; Neto, P. R. C.; Torres, E. A.; Castro, M. P.; Jesus, C. A. C. (2009), Cadeia do biodiesel da bancada à indústria: uma visão geral com prospecção de tarefas e oportunidades para P\&D\&I. Química Nova, 32, 793-808.

Shay, E.G. (1993),Diesel fuel from vegetable oils: status and opportunities. Biomass and Bioenergy, 4, 227-242.

Soares, C. M.; Itavo, L. C. V.; Dias, A. M.; Arruda, E. J.;Delben, A. A. S. T.; Oliveira, S. L.; Oliveira, L. C. S. (2010), Forage turnip, sunflower, and soybean biodiesel obtained by ethanol synthesis: Productionprotocols and thermal behavior. Fuel, 89, 3725-3729.

Tang, H.; De Guzman, R. C.; Simon, K. Y.; Salley, S. O. (2010), Effect of Antioxidants on the Storage Stability of Soybean-Oil-Based Biodiesel. Energy Fuels, 24, 2028 - 2033. 
